\title{
Metallographic Examination of Bronze Bracelets from Hasanlu
}

\author{
George F. Vander Voort
}

Buehler Ltd, 41 Waukegan Road, Lake Bluff, Illinois, 60044 USA

Hasanlu is an early Iron Age settlement located in northwestern Iran. It dates back to the second millennium B.C., 1450 B.C., until it was destroyed around 800 B.C. Although the site attracted the attention of the British archeologist Sir Aurel Stein due to artifacts recovered from burial mounds in the 1930s, it was not given substantial attention until the discovery of the "Hasanlu Golden Bowl" in 1958. The Hasanlu archeology project began in 1957 and was greatly stimulated by this discovery. It ended in 1977. The excavations were sponsored by The University of Pennsylvania Museum and the Metropolitan Museum of Art of New York in cooperation with the Archeological Service of Iran.

The author obtained six specimens from the University of Pennsylvania Museum. The six specimens consisted of three cast and three wrought bronze bracelet sections, as defined in Table 1. The three cast specimens were from the same bracelet.

Table 1. Chemical Analysis of the Bracelets (weight percent)

\begin{tabular}{|c|c|c|c|c|c|c|c|c|c|}
\hline No. & Specimen Code & $\mathrm{Cu}$ & $\mathrm{Sn}$ & $\mathrm{As}$ & $\mathrm{Fe}$ & $\mathrm{Pb}$ & $\mathrm{Ag}$ & $\mathrm{Ni}$ & $\mathrm{Sb}$ \\
\hline 1 & HAS 60-617 & 87.2 & 9.62 & 0.221 & 0.202 & 0.443 & 0.114 & 0.150 & 0.088 \\
\hline 2 & HAS 59-545 & 90.3 & 8.82 & 0.057 & 0.143 & 0.244 & 0.122 & 0.133 & 0.091 \\
\hline 3 & HAS 60-1036a & 90.3 & 7.22 & 0.182 & 0.215 & 0.518 & 0.261 & 0.115 & 0.113 \\
\hline 4 & HAS 59-843a & 86.0 & 12.5 & 0.203 & 0.155 & 0.603 & 0.077 & 0.108 & 0.149 \\
\hline
\end{tabular}

For simplicity, they will be referred to as specimens 1,2,3 and 4. As there are three specimens of the cast bracelet in mount 1 (HAS 60-617), they will be referred to by their size. The chemical analysis shows that they are similar in composition although the tin content of the fourth specimen is somewhat higher than the other three.

\section{Cast Bracelet Specimens}

There were three in the mount and they will be referred to as large, medium and small based on their size. All contained shrinkage cavities and their surfaces were corroded due to years of burial, Fig. 1. All of the specimens exhibited substantial surface corrosion, which was often (in the wrought specimens) along the grain and twin boundaries (see Fig. 2). The wrought structures were all annealed after forming as evidenced by their equiaxed, twinned grain structures, Figures 2 and 3. Not shown in the abstract is the substantial corrosion attack in the wrought specimens, particularly number 4 . Figure 3 (right) shows the extreme center of this specimen and it can be clearly seen that even at low magnification, the entire field of view cannot be filled with non-corroded matrix microstructure. The interior microstructure, revealed by color etching with Klemm's III, shows that the grain size of bracelet specimen number 4 was much finer than that of wrought bracelet specimens 2 and 3. But, this study shows that the metallurgists at Hasanlu had an understanding of hot working and annealing. 

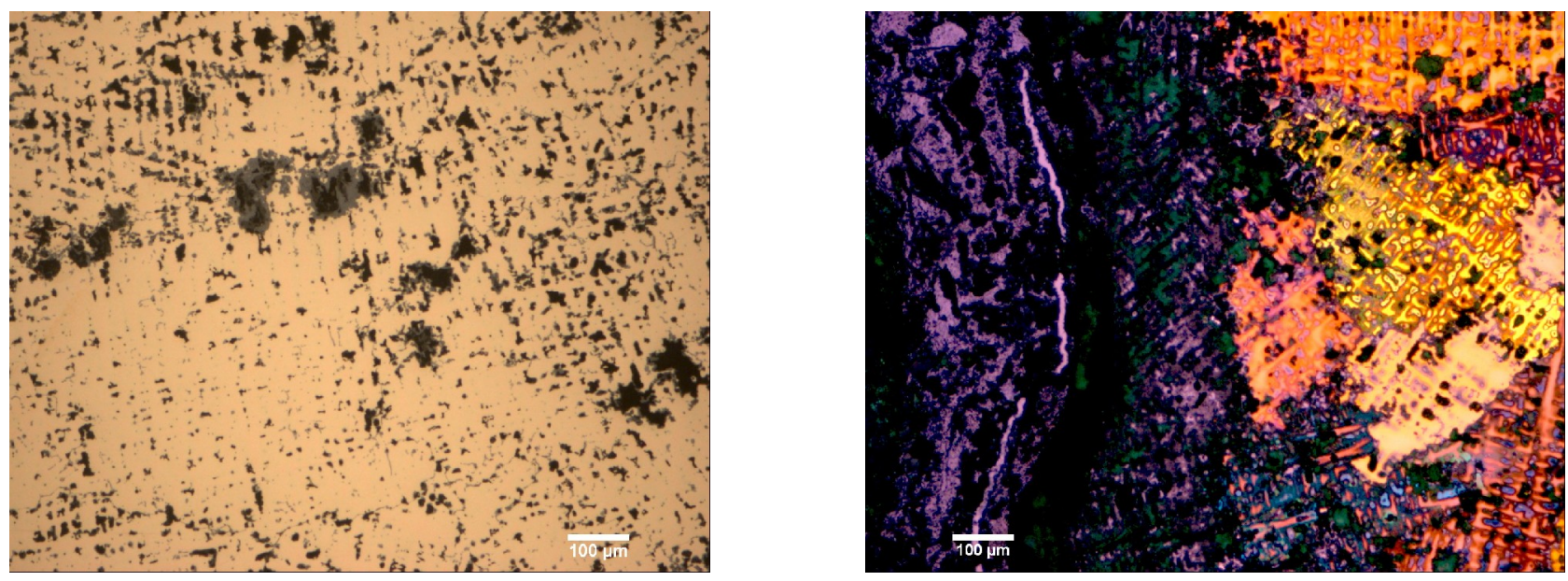

Figure 1. As-polished and color etched (Klemm's III) appearance of the interior (left) and surface dendritic microstructure (right) of the largest as-cast specimen.
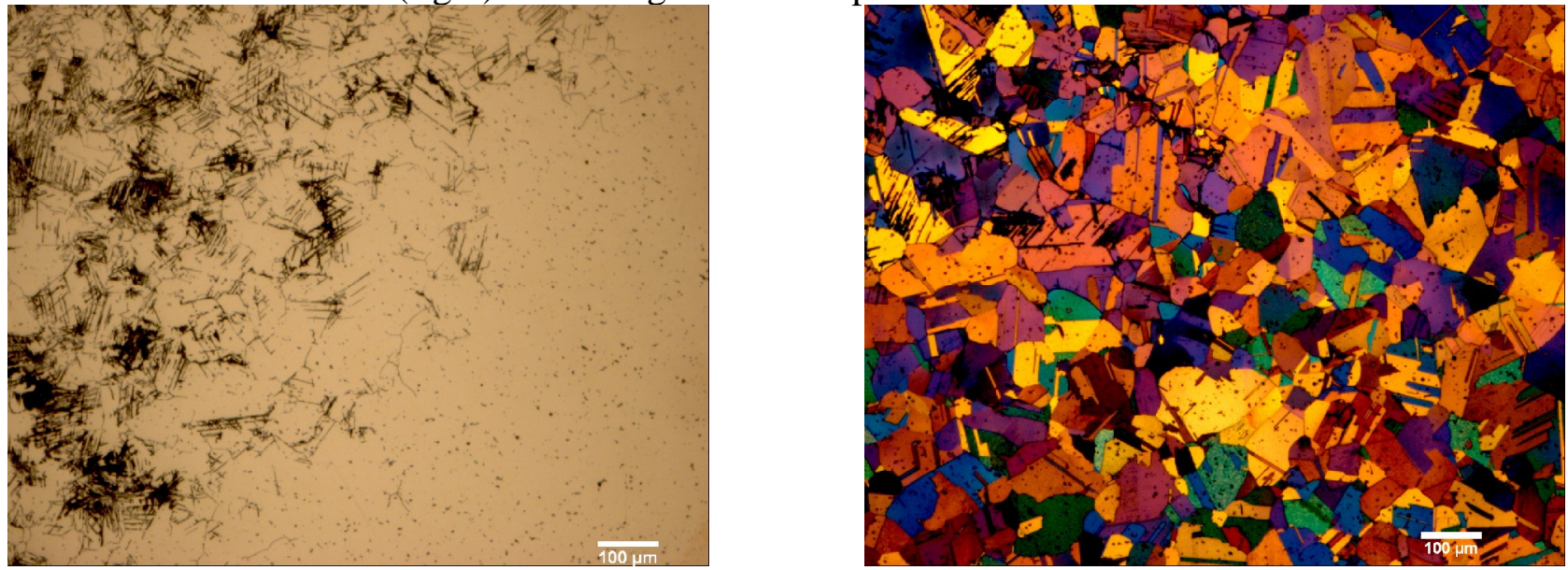

Figure 2. As-polished and color etched (Klemm's III) appearance of the surface corrosion attack (left) and the recrystallized interior grain structure (right) of wrought specimen 2.
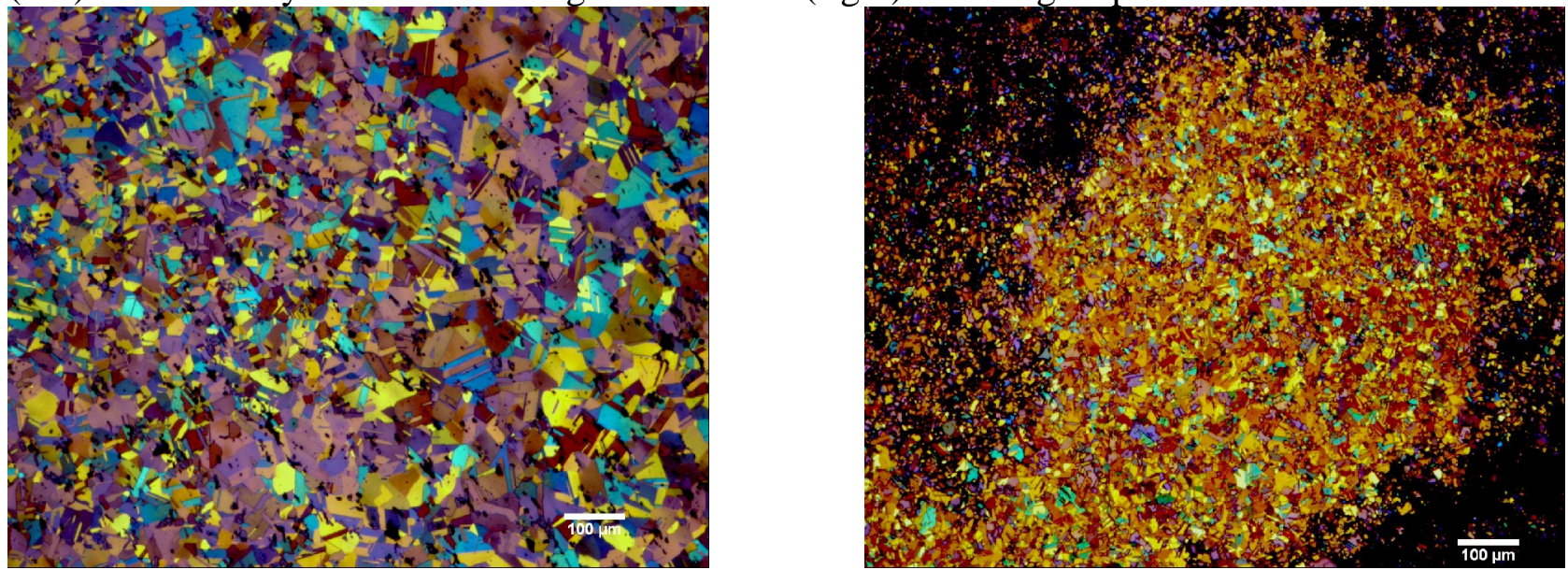

Figure 3. Interior microstructures of wrought specimens 3 (left) and 4 (right). 